doi:10.5937/jaes13-6909

Paper number: 13(2015)2, 314, $71-78$

\title{
APPLICATION OF EMBEDDED CONDITION MONITORING SYSTEMS IN PALLET INDUSTRY
}

\author{
Dr Miloš Milovančević* \\ University of Niš, Faculty of Mechanical Engineering, Niš, Serbia \\ Dr Boban Anđelković \\ University of Niš, Faculty of Mechanical Engineering, Niš, Serbia \\ Dr Jelena Stefanović-Marinović \\ University of Niš, Faculty of Mechanical Engineering, Niš, Serbia \\ Ljubomir Vračar \\ University of Niš, Faculty of Electronic engineering, Niš, Serbia
}

The goal of the predictive condition monitoring of planetary power transmissions in pellet mills is to indicate deterioration in the condition of the power transmissions before the actual failure occurs. In many cases, the defect does not cause an immediate interruption of the process. If this is the case, the defective part can be replaced or repaired during normal, scheduled maintenance periods, provided that the defect has been found in a sufficiently early stage. The most common causes of failure of rotary machines are faults in bearings, the stator and the rotor. There are many methods for the predictive condition monitoring of rotary machines. The analysis can be based on different measured quantities. These include, for example, temperature, current, magnetic flux density and vibration. The basic design idea is to create a measurement and data collection system for condition monitoring in which the data analyses and decision-making are based on fuzzy logic programming. In this paper, a low-cost optimal micro configuration for measurement and condition monitoring of data collection system of pellet mills power transmission is presented. The system is based on PIC (Programmable Interface Controller) microcontrollers and represents the complete solution for condition monitoring regarding vibration, temperature and rpm measurement. The microcontroller based system also has an integrated function with control application based on fuzzy logic.

Key words: Vibrations, Condition monitoring, Fuzzy logic

\section{INTRODUCTION}

The data collection system for a pellet mill consists of several sensor units attached to data-collecting units. There can be one or more collector units all having one or more sensor units connected. In order to keep the sensors inexpensive, they are based on a simple microcontroller with no external memory. Therefore, the collector units provide a temporary storage for the measurement data. If a particular measurement generates data more than the internal RAM of the microcon 7 troller can store, the data transmission between the sensor unit and the collector unit has to be in real time. However, the data transmission from the collector unit onward can be slower and can be packet-based.

The advantages of a multi channel collector unit realization include a simple communications protocol, because each transmission line has only one communicating pair, the collector unit and the sensor unit. Furthermore, the interface between the sensor unit and the transmission cable can be made fixed (i.e. con ᄀnector-less), thus making the connection both more reliable and less expensive. In the case of the bus based realization, the connections can be made fixed too, but this makes it very difficult to modify an existing system. Moreover, in the case of the multi channel collector unit, the whole system is more reliable, since a defect in one spot of the cable cancels the connection between the collector unit and one sensor unit only, instead of, in the worst case, making all of the sensors unavailable to the collector unit.

A sensor unit with the capability of measuring acceleration and temperature was developed. The unit was based on Anaᄀlog Devices' ADXL311 micro machined accelerometer. The ADXL311 was found suitable for bearing condition moni-

* University of Niš, Faculty of Mechanical Engineering, Aleksandra Medvedeva 14, 18000 Niš; 
toring when vibration levels were not particularly high, within the region of $\pm 5 \mathrm{~g}$. The acceleration signal was sampled at a frequency of $20 \mathrm{kHz}$ and digitized using 12 bits [05, 09].

The length of one vibration measurement was set to 32768 samples, and each sample was padded to 16 bits. It could be calculated that the space needed to store one acceleration measurement was 64 kilobytes, which was far more than the capacity of the internal SRAM of the microcontroller. Therefore, the measured data needed to be transferred to the collector unit in real time. The minimum acceptable transfer rate could be calculated when the sampling frequency and the length of a sample were known. With a sam $\neg$ pling frequency of $20 \mathrm{kHz}$ and 16 bits per sample, this was 320,000 bps.

\section{PIC MICROCONTROLLER APPLICATION}

PIC is a family of Harvard architecture microcontrollers made by Microchip Technology, derived from the PIC1640 originally developed by General Instrument's Microelectronics Division. The name PIC initially referred to "Programmable Interface Controller".

The collector unit features the Microchip PIC16F877A microcontroller with 64 kilo words of 16-bit wide external SRAM memory. This memory is used to store the measurement data fetched from the sensor units. Its capacity is sufficient to hold two of the previously described vibration measurements at a time.

The PIC architecture is distinctively minimalist. It is characterized by the following features:

- Separate code and data spaces (Harvard architecture);

- A small number of fixed length instructions;

- Most instructions are single cycle executions (4 clock cycles), with single delay cycles upon branches and skips;

- A single accumulator (W), the use of which (as source operand) is implied (i.e. is not encoded in the opcode);

- All RAM locations function as registers of both source and/or destination of math and other functions;

- A hardware stack for storing return addresses;

- A fairly small amount of addressable data space (typically 256 bytes), extended through banking;
- Data space mapped CPU, port, and peripheral registers;

- The program counter is also mapped into the data space and writable (this is used to implement indirect jumps).

Unlike most other CPUs, there is no distinction between memory space and register space because the RAM does the job of both memory and registers, and the RAM is usually just referred to as the register file or simply as the registers.

\section{Data acquisition process}

The collector unit acts as a master on the sensor bus. Only by its request can a sensor unit start sending data. The collector unit initiates the data transfer by sending an atten-tion sequence followed by the number of the sensor the request is addressed to and the number of the measurement wanted. After sending the request, the collector unit starts to listen for incoming data from the sensor bus and store it in the memory. When there is a pause long enough in the in 7 coming data or the transmission has exceeded a specified time limit, the collector unit deems the transmission fin 7 ished. The number of the sensor and the measurement as well as the start address of the storing of the measurement can be set by the user of the collector unit. The amount of data the measurement should generate can also be set, which makes it possible for the collector unit to judge whether the measure $\urcorner$ ment is successful or not. On the whole, the collector unit is versatile in the sense that it can be used to retrieve data from many different types of sensors or other devices [02].

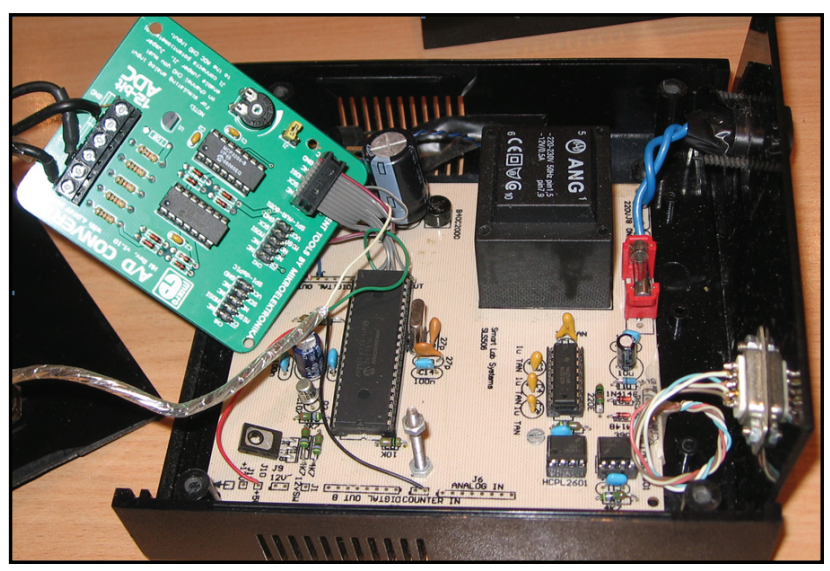

Figure 1: Optimal PIC based monitoring system

There are several possibilities to realizing communications from the collector unit to a higher level in the information infrastructure. Installing 
new cables is not usually economically feasible. Instead, wireless communications or exרisting infrastructure, such as field buses or power cabling, can be used. In the developed system, the physical link was im?plemented with a similar RS-232 connection as in the case of the sensor bus. A photograph of the newly developed collector unit is shown in Figure 1.

The optimal micro system shown in Figure 1 is presented disassembled. The system has a main board, where microcontroller is imbedded. A small electronic board with ADC is added and connected to the main board through the SPI communication protocol.

The optimal micro configuration based on PIC microcontroller is created as a complex integrated system in Figure 2. Condition monitoring based primarily on vibration but also on temperature measuring is not the only function as it can be observed from the algorithms. The microcontroller is programmed to simultaneously collect data from sensors and to control relays in order to obtain full pellet mill working order control based on fuzzy logic [03, 04].

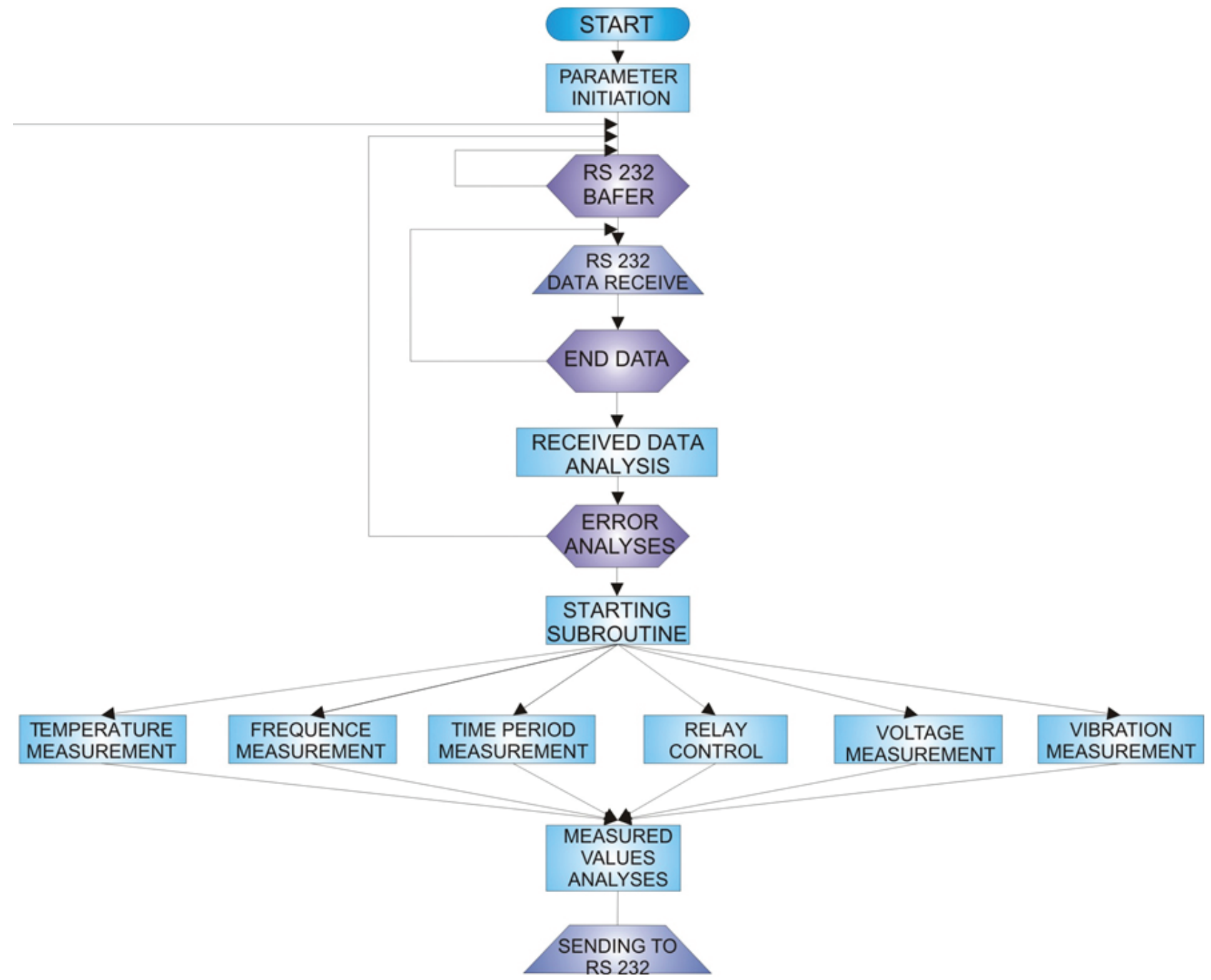

Figure 2: PIC initiation algorithm

\section{PELLET MILLS CONDITION MONITORING}

Wood pellets are a type of wood fuel, generally made from compact sawdust. They are usually produced as a by-product of sawmilling and other wood transformation activities. The pellets are extremely dense and can be produced with a low humidity content (below 10\%) that allows them to burn with very high combustion efficiency.
Further, their regular geometry and small size allow automatic feeding with very fine calibration. They can be fed to a burner by auger feeding or by pneumatic conveying.

Pellets are produced by compressing the wood material which has first passed through a hammer mill to provide a uniform dough-like mass. This mass is fed to a press where it is squeezed 
through a die having holes of the required size (normally $6 \mathrm{~mm}$ in diameter, sometimes $8 \mathrm{~mm}$ or larger). The high pressure of the press causes the temperature of the wood to increase greatly, and the lignin plastifies slightly forming a natural 'glue' that holds the pellet together as it cools.

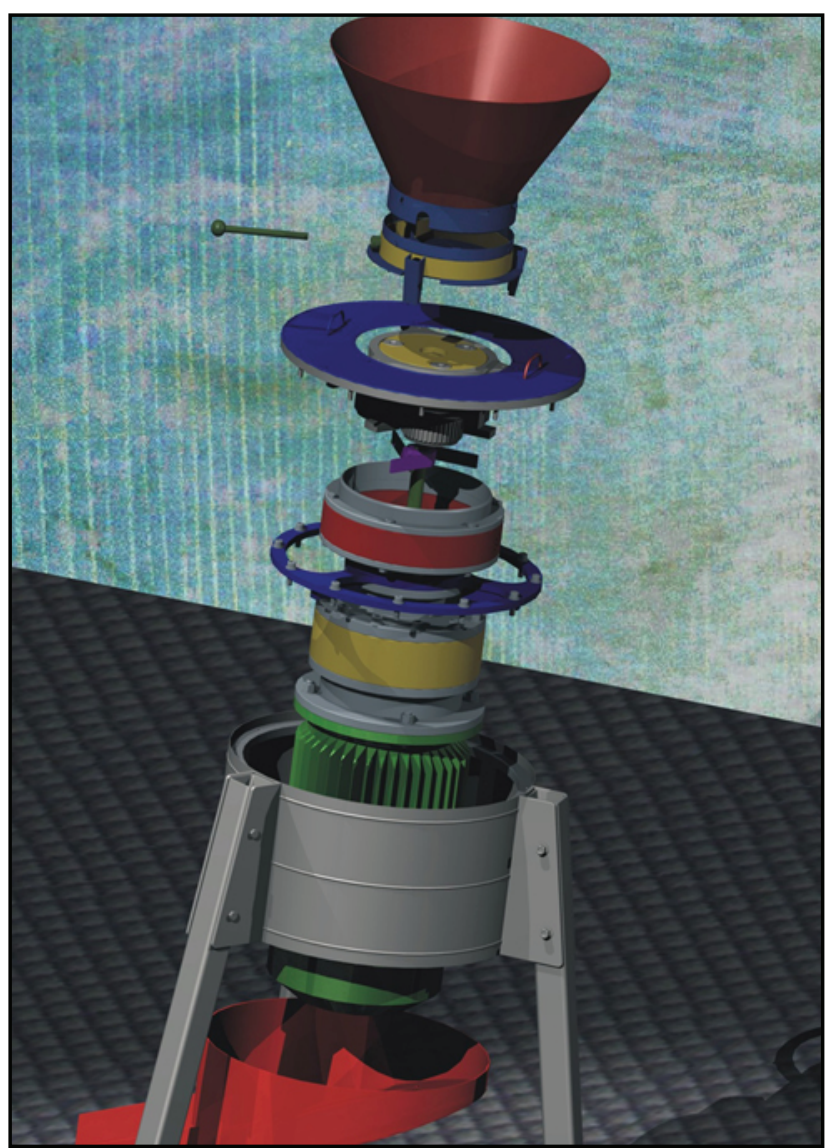

Figure 3: Exploded 3D view of pellet mill

In order to obtain a complete pellet mill monitoring, the optimal micro configuration system (see Figure 1) was developed on a PIC16F877A microcontroller platform. To enhance system resolution from 10-bit to 12-bit, ADC-MCP3204 was added. The system was developed a new both from the aspect of hardware and software at the Faculty of Mechanical Engineering in Niš.

The optimal configuration for vibration monitoring is created by applying axiomatic design postulates to incorporate industry demands in functional requirements and design parameters [08]. Apart from the applied condition monitoring techniques on a subsystem level (die temperature, planetary power transmission vibrations), there is already a lot of information available in the pellet mill. Normally, this information is only used at the level of safeguarding. Thus, temperature monitoring is of importance, since over-heating of die can cause pellets deformation or collapse. Exceeding the alarm levels often simply results in a pellet mill shutdown and the wait for remote restart or repair. By application of more advanced methods of signal analysis, focused on trends of representative signals or combination of signals, significant changes in mill behavior can be detected at an early stage [07, 01].

\section{Fault detection}

Nowadays, condition monitoring of a pellet mill system is restricted to the individual performance of the servo motors themselves at the level of detection of maximum current, as shown in Fig. 4. However, model based condition monitoring of all three servo systems is a promising possibility in this situation. Model based condition monitoring is suitable for non-stationary operation.

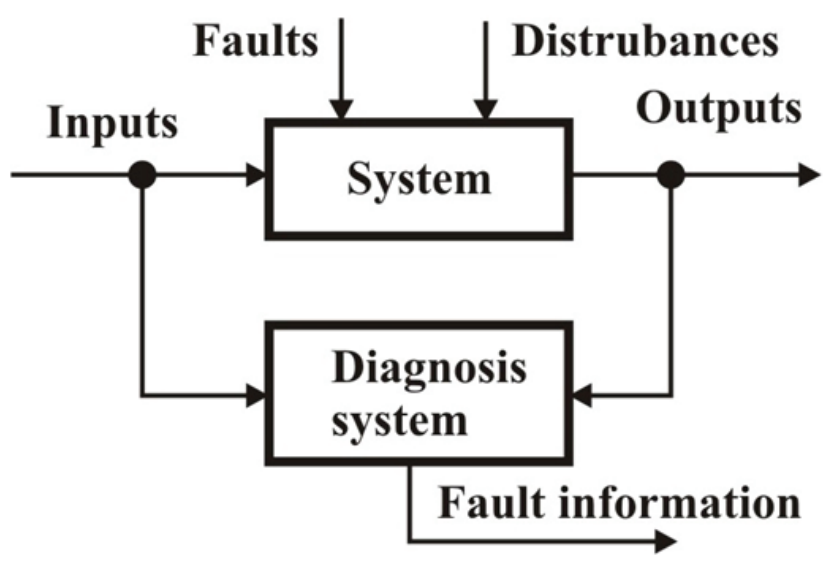

Figure 4: Principle of model based fault detection

The diagnosis can be based on the residual of the process and estimator output signals (see Figure 5). In this situation, a constant model is used. The difference between the output of the system and the output of the model can be monitored. A trend analysis of this residual can be used to detect the changing characteristics of the system [06].

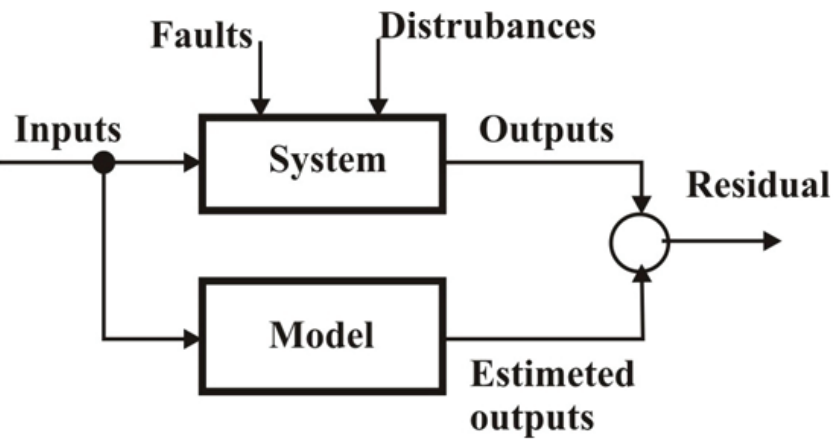

Figure 5: Fault estimation based in residual 


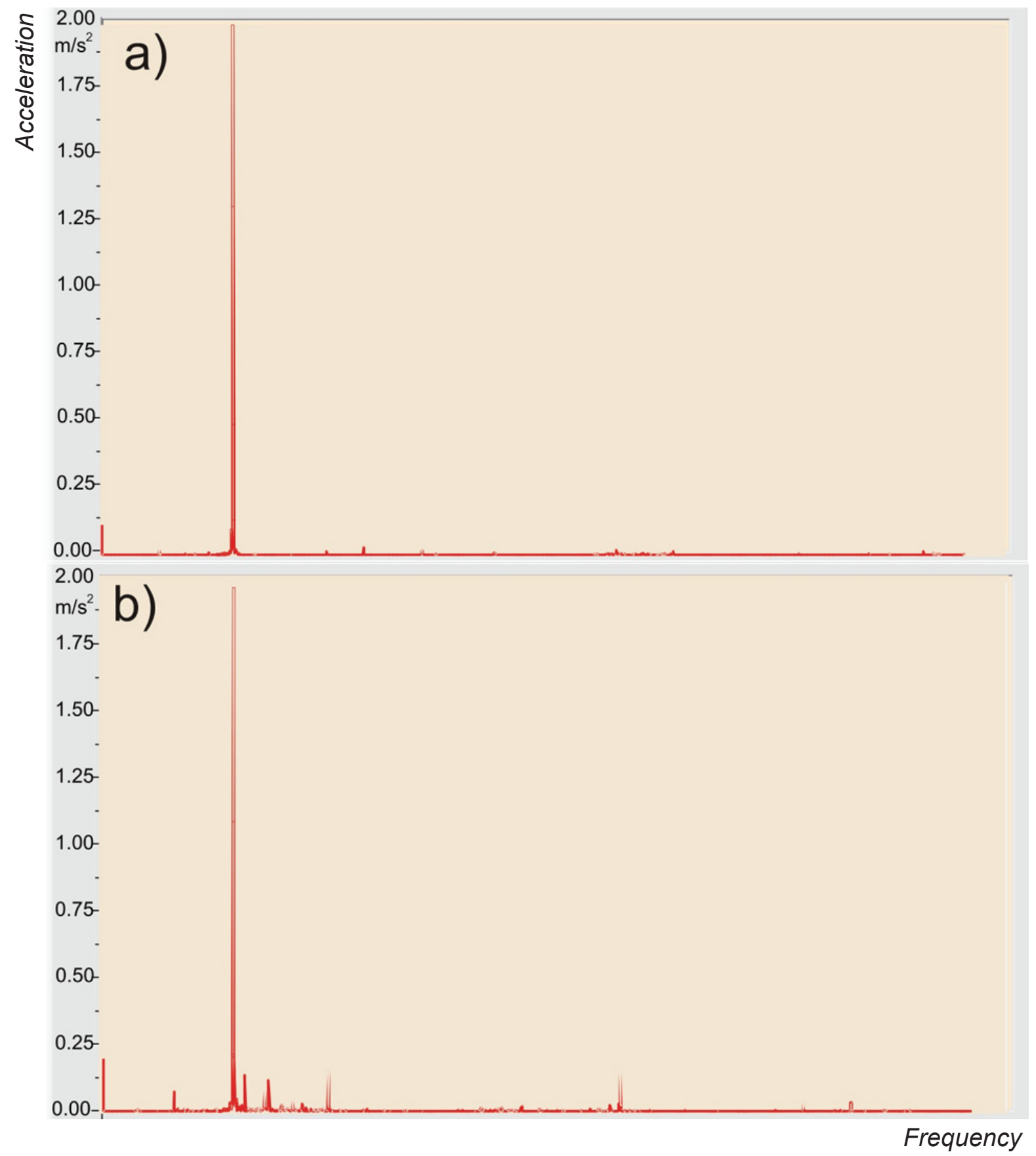

Figure 9: Example of fault detection based on FFT $(a, b)$

The fault detection is often based on frequency analysis and level detection for certain frequency bands. Based on the level of amplitudes, status signals can often be defined and generated. Presented diagrams of fault detection based on FFT analyses are a product of newly developed software for vibration signal acquisition and analyses (Figure 9).

Vibration diagram a) in Figure 7 represents a vibration spectrum of the planetary gear transmission in regular working order. Wood feeding to press was automatically controlled during vibration monitoring. Vibration diagram b) is a vi- bration spectrum that is different from vibration spectrum a) because wood moisture content has changed. Vibration spectrums c) and d) were captured while wood feeding of the press was switched off. At first, vibration spectrum c) showed a wide vibration spectrum with amplitudes of up to $1,75 \mathrm{~m} / \mathrm{s}^{2}$. After a certain period of time, the vibration spectrum narrowed and amplitudes were up to $3,5 \mathrm{~m} / \mathrm{s} 2$, with the die temperature also increasing by $15 \%$. During the data acquisition, rotation velocity was constant. Thus, without the rotation regulation, the production process is inefficient. 


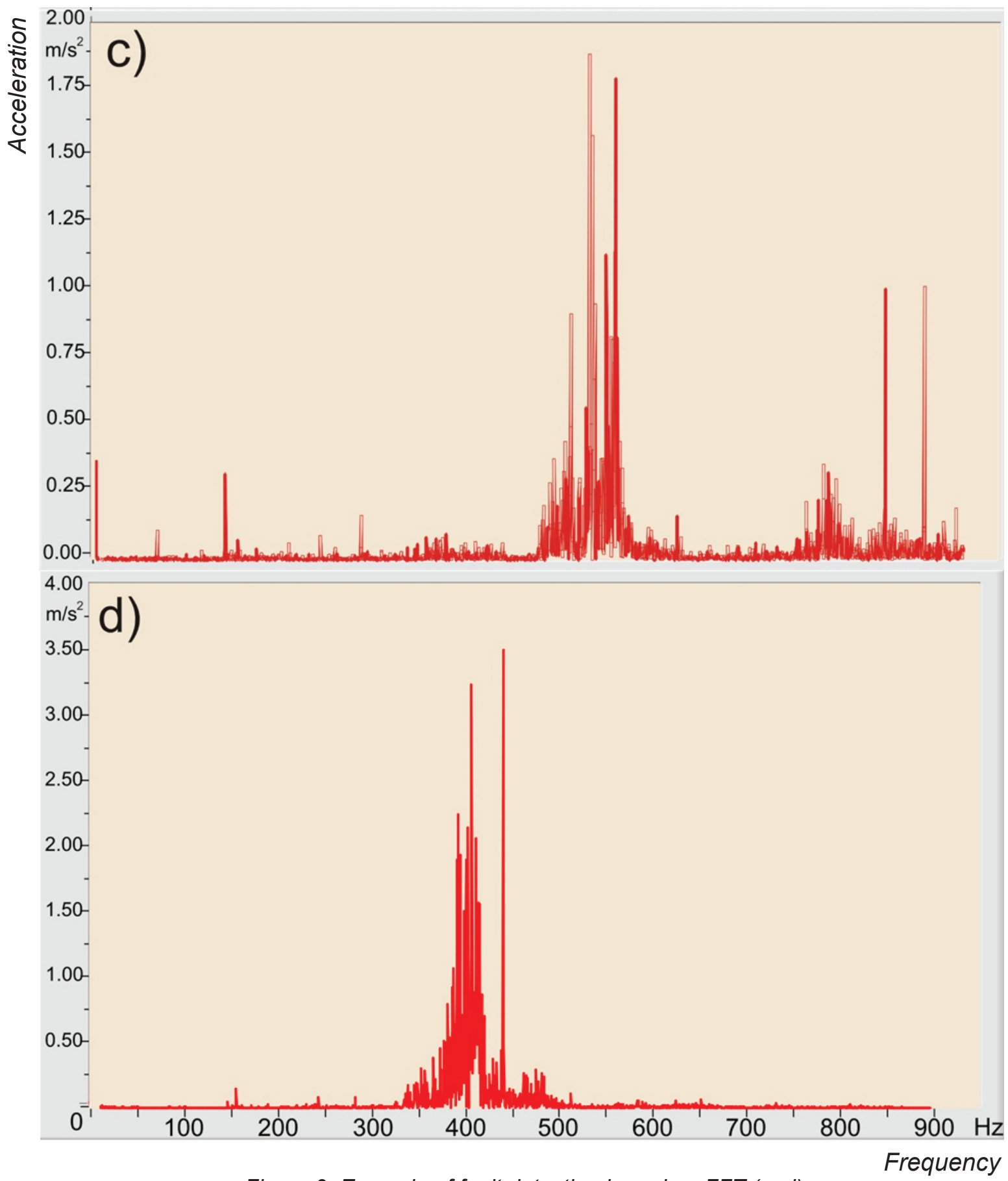

Figure 9: Example of fault detection based on FFT $(c, d)$

The rotation velocity regulation in relation to input parameters is complex. The regulation is based on the idea that vibration acceleration and temperature fluctuations are controlled by constant rpm variations. Software for data acquisition and signal analyses was created and customized for planetary gear vibration monitoring. The analyses of pellet mill exploitation and the results of vibration monitoring using the newly developed configuration proved that strict industry requirements could be fulfilled completely by applying PIC technology.

\section{CONCLUSION}

The embedded system based on PIC microcontroller condition monitoring can be applied successfully for pellet mill monitoring and control. The integration of functional requirements, design parameters and industry demands resulted in the creation of a new software and hardware for pellet mills safeguarding. In other industries, condition monitoring provisions are normally separate systems, apart from the machine control and safeguarding functions. In this paper, 
the pellet mills monitoring was focused on an embedded, a microcontroller based integrated functional control system developed on fuzzy logic. The application of MEMS accelerometers and PIC microcontrollers in pellet mills condition monitoring has an important roll in expanding the product life cycle as well as in reducing production costs. Application of embedded system in pallet mills has proven to be possible in the sense of gear transmission vibration and die temperature monitoring. Also, fuzzy logic control of pallet mill based on vibration and temperature in relation to main shaft rotation is possible. The effectiveness of these systems is not yet evident, since prototype testing is completely different from exploitation tastings. Due to the non stationary operation, it appears to be difficult to develop effective algorithms for mills early fault detection, especially for variable speed operations. Practical experience builds up very slowly, because component degeneration is a slow process, and additional information about mill transmission gear loads and operational conditions is only fragmentarily available. Further research is this particular project is focused on integration of vibration/temperature monitoring, rotation and wood feed control. Embedded systems research in the first faze, is going to be oriented to further development of single chipset monitoring systems based on PIC technology and in the second faze energy harvesting in the function of mechanical systems condition monitoring.

\section{REFERENCES}

1) ADXL311 Data Sheet", Analog Devices, [Online] Available: http://www.analog.com/ media/en/technical-documentation/obsoletedata-sheets/ADXL311.pdf

2) Čudina, M. , 2003, Detection of cavitation phenomenon in a centrifugal pump using audible sound, Mechanical System and Signal Processing, Vol. 17, No. 6, pp. 1335-1347.

3) Čudina, M., Prezelj, J., 2008, Use of audible sound for safe operation of kinetic pumps, International Journal of Mechanical Science. Vol 50, No. 9, pp. 1335-1343.

4) Čudina, M., Prezelj, J., 2009, Detection of cavitation in operation of kinetic pumps, use of discrete frequency tone in audible spectra. Applied Acoustics. Vol. 70, No. 4, pp. 540-546.

5) Matić N., Andrić D., 2000, PIC mikrokontroleri, Mikroelektronika Beograd.

6) MCP6022 Data Sheet", Microchip Tecnology Inc, [Online] Available: http://ww1.microchip. com/downloads/en/DeviceDoc/21685d.pdf

7) Milenković D., 1988, Nestabilno strujanje kroz kola turbomašina izazvano globalnim gubitkom stabilnosti, 18. jugoslovenski kongres teorijske $\mathrm{i}$ primenjene mehanike, Vrnjačka Banja, str. 320-326.

8) Milovančević M., Stefanović Marinović J., Anđelković B. Veg A., 2010, Embedded condition monitoring of power transmission of a pellet mill. Transactions of Famena XXXIIII2 (2010), Faculty of mechanical engineering and naval architecture, pp. 71-79, Zagreb

9) Milovančević M., Veg A.,2009, Application of axiomatic design on vibro-diagnostic system, 9th International Conference "Research And Development In Mechanical Industry" RaDMI, p.p. 295-301, Serbia.

Paper sent to revision: 09.10.2014.

Paper ready for publication: 15.06.2015. 